\title{
\begin{tabular}{l|l} 
Mibraries & DSpace@MIT
\end{tabular}
}

MIT Open Access Articles

Variation in Lightning Simulations to Assess Grounding Safety of Corrugated Stainless Steel Tubing (CSST)

The MIT Faculty has made this article openly available. Please share how this access benefits you. Your story matters.

As Published: https://doi.org/10.1007/s10694-019-00885-x

Publisher: Springer US

Persistent URL: https://hdl.handle.net/1721.1/131870

Version: Author's final manuscript: final author's manuscript post peer review, without publisher's formatting or copy editing

Terms of use: Creative Commons Attribution-Noncommercial-Share Alike 


\section{Variation in Lightning Simulations to Assess Grounding Safety of Corrugated Stainless Steel Tubing (CSST)}

Cite this article as: Bryan Haslam and Thomas W. Eagar, Variation in Lightning Simulations to Assess Grounding Safety of Corrugated Stainless Steel Tubing (CSST), Fire Technology https://doi.org/10.1007/s10694-019-00885-x

This Author Accepted Manuscript is a PDF file of an unedited peer-reviewed manuscript that has been accepted for publication but has not been copyedited or corrected. The official version of record that is published in the journal is kept up to date and so may therefore differ from this version.

Terms of use and reuse: academic research for non-commercial purposes, see here for full terms. https://www.springer.com/aam-terms-v1 
Title: Variation in Lightning Simulations to Assess Grounding Safety of Corrugated Stainless Steel Tubing (CSST)

Authors: Bryan Haslam and Thomas W. Eagar

Affiliation: Massachusetts Institute of Technology

Address: 77 Massachusetts Ave 4-136, Cambridge MA 02139

Corresponding Author's Email: bhaslam@mit.edu 
Fire Technology manuscript No.

(will be inserted by the editor)

\title{
Variation in Lightning Simulations to Assess Grounding Safety of Corrugated Stainless Steel Tubing (CSST)
}

immediate

the date of receipt and acceptance should be inserted later

\begin{abstract}
Codes and standards for lightning often rely on simulations due to the difficult nature of lightning testing, as in the case of corrugated stainless steel tubing (CSST). A small set of simulations by CSST manufacturers were previously used to justify the suggestion that grounding CSST would make it safe from perforation in the presence of lightning. Such a small set of simulations does not account for the uncertainty of lightning and the situations where it may interact with CSST. We account for these uncertainties in this work by performing thousands of simulations that use different combinations of simulation parameters. For example, for one scenario we run 2560 simulations with a variety of different waveforms and different impedance values. The waveforms follow IEC 62305 with rise times ranging from 0.25 to $10 \mu \mathrm{s}$ and fall times ranging from 100 to $1000 \mu s$ and the impedance values were varied by $+/-25 \%$. Our results show that there are cases where grounding may prevent perforation, cases where grounding may reduce the damage but not prevent perforation and cases where grounding increases the chances of perforation. Our results further show that for lightning strikes with peak current greater than the median, there was never a case where grounding could have prevented perforation. Our methods provide a way to perform more comprehensive simulations to replicate what may happen in nature and better inform decisions made about codes and standards. In particular we show grounding of CSST will not prevent fires when assaulted by lightning with any reasonable degree of certainty.
\end{abstract}

\section{Introduction}

Lightning is a powerful electrical discharge that transfers an enormous amount of energy and can cause significant damage to property and people [20]. Damage includes fire or injury and in severe cases total loss of property or death [35]. It is estimated that lightning results in $\$ 333$ million to $\$ 1$ billion in insurance costs per year [35]. It is further reported that between 1959 and 1994 there were 9,818 injuries and 3,239 fatalities cause by lightning in the United States [8]. Safety codes and standards are created to ensure measures are implemented to help prevent such damages and injuries. For example, IEC 62305 [18] and NFPA 780 [23] set standards for lightning protection of structures, property and people. Products that may cause damage by interacting with lightning must also follow codes and standards to ensure safe operation.

Ideally lightning protection codes and standards would be based on physical tests showing safe behavior of products when interacting with actual lightning strikes, but it is challenging to perform physical tests with real lightning. Lightning produces powerful and unique combinations of voltage, current and duration - any two of which can be replicated in a laboratory, but only nature can replicate all three parameters in the same flash.

It is nearly impossible to predict when and where natural lightning will strike. Triggered lightning is feasible, but still unpredictable and is very expensive [36]. Laboratory experiments are controlled and repeatable, but cannot replicate the power found in a lightning strike. In practice, researchers must rely on computer simulations to infer what might happen in the case of a lightning strike. Simulations are setup by measuring (1) characteristics of lightning strikes, such as peak current, and (2) characteristics of the materials the lightning will interact with, such as impedances of conductors in a structure. These values are used as parameters in the simulations. What is often overlooked or ignored is that fact that only some

Address(es) of author(s) should be given 
fraction of measurements can be made and the rest of the simulation parameter values must be assumed or inferred. The vital scientific question is if the simulations adequately represent what can happen in reality. In this paper we explore which components of lightning simulations are assumed or inferred and what effect changes in those assumptions would have. That information allows us to determine if the results can be extended to what occurs in nature and how the results of these simulations should be used for safety codes and standards.

In this paper in particular, we focus on fire safety of corrugated stainless steel tubing in the presence of lightning. This topic is important because there is approximately 1 billion feet of the original yellowjacketed CSST product in the United States and the safety of this product has garnered the attention of numerous Fire Marshals, the National Fire Protection Agency (NFPA) and even the United States Senate [37]. It is universally recognized that lightning can create holes in yellow-jacketed corrugated stainless steel tubing (CSST) found in homes, which results in gas leaking from the pipes, the gas igniting and causing a house fire [1]. CSST originally received LC1 approval in 1991 [2] and is preferred over traditional black iron pipe to carry gas in homes because it reduces installation time and requires fewer fittings. This convenience is possible because CSST has a much thinner wall (approximately $0.25 \mathrm{~mm}$ compared to the $2.5 \mathrm{~mm}$ of black iron pipe), but that also makes it more susceptible to perforation when assaulted by lightning. After numerous fires blamed on CSST in the presence of lightning, CSST manufacturers began marketing a conductive black jacketed CSST (compared to the insulative yellow-jacketed CSST) in 2005 which was designed to be less susceptible to lightning damage [30]. To address the remaining 1 billion feet of original yellow jacketed CSST, manufacturers suggested a requirement to ground the CSST to the electrical earth ground with a bonding wire. In 2009 the National Fire Protection Agency (NFPA) adopted this requirement into the Fuel Gas code [22, 19], but asked for further evidence showing that bonding would indeed prevent fires. A large part of the basis for the fuel code change was computer simulations [14, 33].

These codes and standards, and therefore the safety of people's lives and homes, rely on computer simulations. Ensuring that the simulations are adequate is a vital part of adopting these codes and standards. Previous work, including lab tests and computer simulations, have concluded that although a bonding wire may be sufficient in some situations, it is not sufficient in most cases $[10,15]$. In this paper we build on previous work by identifying several considerations that influence results and ways the assumptions of the simulations affect the simulation outcomes. We share our findings and explain what factors need to be taken into account when performing lightning circuit simulations.

The paper is organized as follows. We present a brief overview of lightning and the problem of perforation of CSST, then provide an overview of simulations and failure analysis. We then outline the simulation methods used and describe what factors most significantly affect simulation outcomes including choice of electrical circuit elements, lightning waveform shape and modeling of uncertainty. We then show how different simulation parameters, particularly for lightning waveforms, affect the simulation outcomes. We then provide a discussion of the parameters we found most likely to influence simulation outcomes and how simulations should be used. We conclude by suggesting guidelines for performing simulations that should be used to ensure safe application of simulation results to codes and standards.

\section{Background}

\subsection{Lightning}

The physics of lightning has been detailed in several reference works: [20],[29] with the following article providing a concise background: [36]. The study and measurement of lightning has progressed significantly over the 250 years since Benjamin Franklin performed early studies [34]. Recent data not found in classic lightning textbooks shows that lightning is more powerful than previously believed with the strongest $1 \%$ of strikes having a peak current of $150 \mathrm{kA}$ and a total charge up to 200 Coulombs (C) for a negative strike and $500 \mathrm{kA}$ and $700 \mathrm{C}$ for a positive strike [11]. It is important to have this updated information so that the power of lightning is not underestimated.

\subsection{Fire from CSST and Lightning}

Lightning current can enter a structure through a variety of electromagnetic phenomena and will preferentially flow through electrical conductors such as metals [4]. Metal conductors in homes include wires, ducts, pipes, railings, appliances and so forth. Electrical potential, or voltage, when large enough causes 
an arc to such a conductor. If an arc forms between CSST and another metal conductor, a hole may form in the CSST. Whether or not a hole forms depends on the amount of charge that moves through the arc. In the case of yellow-jacketed CSST, the insulative jacket can concentrate the arc current through a small hole in the jacket resulting in more charge through a smaller area, which in turn results in higher localized heating and increases the likelihood of creating a hole. We have done testing to confirm this phenomena [26] and the manufacturers of CSST have performed similar tests of the threshold current for perforation with similar results - holes are possible with as little as $0.1 \mathrm{C}$ [1].

It was experimentally demonstrated in previous work that when an electrical arc creates a hole in CSST the escaping gas is ignited [15]. Using high speed photography we observed that the spatter from the molten steel ignites the escaping gas. Then as long as the escaping gas is in a confined space such as a wall or attic where CSST is typically located, the flame will continue to burn. It is often the case that a fire will spread quickly so that the original flame is not observed, but we have seen multiple field cases where a flame was visible as gas escaped a hole in CSST immediately after a lightning strike and no other ignition sourcef was near by. These observations were possible because the fires were small and were identified quickly so they did not spread and obliterate the evidence of the ignition sequence. Our experimental work and these field examples show that lightning can cause a hole in CSST, that the escaping gas can be ignited and that the fire can be sustained. Many of the manufacturers dispute this sequence, particularly whether the ignition can be sustained.

For a hole to be created in the CSST, two conditions must be met: (1) an arc is formed when there is enough voltage between two conductors and (2) a hole is formed when enough charge moves across the arc. Regarding (1), it has been shown previously that yellow CSST has a breakdown voltage of $25 \mathrm{kV}$ [14], which is the voltage necessary to create an arc. This measurement was taken in a controlled laboratory setting, but can be treated as an approximation. Given that a lightning strike can have a potential of more than 50 MV (2,000 times the breakdown voltage of the CSST), an arc is relatively easy to achieve [28]. The voltage to create an arc can come directly from the lightning strike or from the lightning current inducing a voltage as lightning current flows along a path of conductors with inductance. Such conductors include electrical wiring, CSST and many other conductors found in a home. Regarding (2), holes have been caused by as little as $0.1 \mathrm{C}$ of charge, that can be used as a threshold for what would cause a fire.

\subsection{Simulations}

In this paper we build on previous work using computer circuit simulations [15]. Circuit models are commonly used in electrical engineering for simulating how a potential circuit will behave in the process of designing a new circuit. Each component in the simulation has characteristic properties modeled by mathematical equations. The circuit elements are connected together (the location of the connections are called nodes) and this creates a set of mathematical equations that the simulation solves to give the voltage at each node and the current through each element. In our simulations, the circuit components represent different metal conductors in a home. When lightning enters a home, different amounts of voltage and current will be found at different locations. The simulation indicates how much voltage can be expected between given points and how much current or charge would flow through an element, including an arc.

For any type of simulation, the user determines what kind of model to use, what model elements to use and what parameters to use for those elements. The software can only solve what the user has setup. The challenge with circuit simulations for lightning is that the following are very difficult to determine: (1) impedances of all potential conductors in a structure, (2) where the lightning current entered the structure and (3) the shape of the lightning current waveform. Some impedances can be measured or estimated. For example, the length of electrical wiring can be measured and the impedance values can be calculated based on the type of wiring. This is not possible for many of the conductors found in a structure. Where the lightning enters the structure may be inferred, but is not possible to know with certainty as an individual lightning strike is unpredictable. To estimate a lightning current waveform, there are tools that can be used to determine the maximum current of a lightning strike, but not the shape of the lightning current waveform or how many Coulombs of charge are in the strike $[6,7]$. In our previous work we showed that changing the impedances or where the location the lightning entered the structure had a large impact on the results of the simulation. In this work we demonstrate how different waveforms assumptions about the lightning waveform can also dramatically affect the simulation outcomes.

We have performed thousands of simulations which taken together can help overcome the uncertainty of an individual lightning event. 


\subsection{Failure Analysis}

We further show that ranges of simulation parameter values should be taken into account. Engineering simulations must account for variability while quality engineering design ensures that failures occur less than an acceptable rate. An acceptable rate is determined by experts based on the severity of the consequences of the failure. A design specification will also include a safety factor, meaning the specification must be more stringent than the original specification by some significant percentage. It is an established principle that the safety factor is applied to the extreme value to be avoided. For example, if someone was building a wall for a nuclear reactor to protect against tsunamis and it was determined that the highest possible predicted tsunami was 10 meters, it would be negligent engineering to build a 10 meter wall and assume that the largest tsunami will only go right up to the top but not over the wall. Instead a safety factor of $50-100 \%$ would be factored in and the wall would be built 15-20 meters tall. The safety factor is applied to the extreme value, not another value such as the mean or median. If the median tsunami was 4 meters, then a safety factor of 50-100\% would suggest building a wall up to of 6-8 meters and would not protect against the extremum value (largest tsunami expected).

In performing lightning simulations, it would be inappropriate to design for the median lightning strike. It would be reasonable to take an extreme value (such as the top $5 \%$ or top 1\%) plus a safety factor.

\section{Methods}

To determine the affects of various parameters of lightning simulations, we selected a circuit from a recent actual lightning-induced CSST fire. We then reviewed the literature on lightning waveforms and simulations to find a range of possible lightning parameters and simulation waveforms that are used. We then performed our own simulations adjusting many different simulation parameters to collect data on the various possible combinations.

The primary question was whether or not grounding as instructed in the manufacturers product data sheets would cause the charge through an arc with the CSST to be less than $0.1 \mathrm{C}$ for different possible simulation parameters. It is trivial to find one set of parameters small enough that will show a result where grounding is reduced to a charge below $0.1 \mathrm{C}$ - what matters is if the same result would hold for all practical variations of those parameters. We account for the following variances in our simulations:

1. Different locations the lightning can enter the structure

2. Different impedance values of conductors

3. Different current waveform shapes

4. Different current magnitudes

5. Presence or absence of grounding bond wire

\subsection{Waveforms for lightning simulations}

One of the most important assumptions in setting up a lightning simulation is the shape of the lightning current waveform. IEC 62305 proposes a specification for a waveform for testing. IEC 62305 suggests several rise time and fall time parameter values, which were decided on in 1980 by the International Electrotechnical Commission (IEC) technical committee TC 81 [16]. The values were largely based on the work of Berger and colleagues from 1975 and $1980[16,5,3]$.

The shape of the general waveform can be seen in Figure 1. The waveform is defined by three parameters: the maximum current $i_{\max }$, a $10 \%-90 \%$ rise time $\left(T_{1}\right)$, and the $50 \%$ fall time $\left(T_{2}\right)$. The shape of the waveform will be represented in this paper by the rise and fall times in the form T1/T2. The waveform could be modeled with many different mathematical functions. Two specific functions discussed in the literature are the double exponential and the Heidler equations: [16]

$$
\begin{aligned}
& i=\gamma i_{\max }\left(e^{-\alpha t}-e^{-\beta t}\right) \\
& i=\gamma i_{\max } \frac{(\alpha t)^{10}}{1+(\alpha t)^{10}} e^{-\beta t}
\end{aligned}
$$

To translate the waveform parameters from $i_{\max }, T_{1}$ and $T_{2}$ to the equation parameters $i_{\max }, \gamma, \alpha$ and $\beta$ we used a custom nonlinear numerical solver. Some typical values are included in Table 1 . The table also 


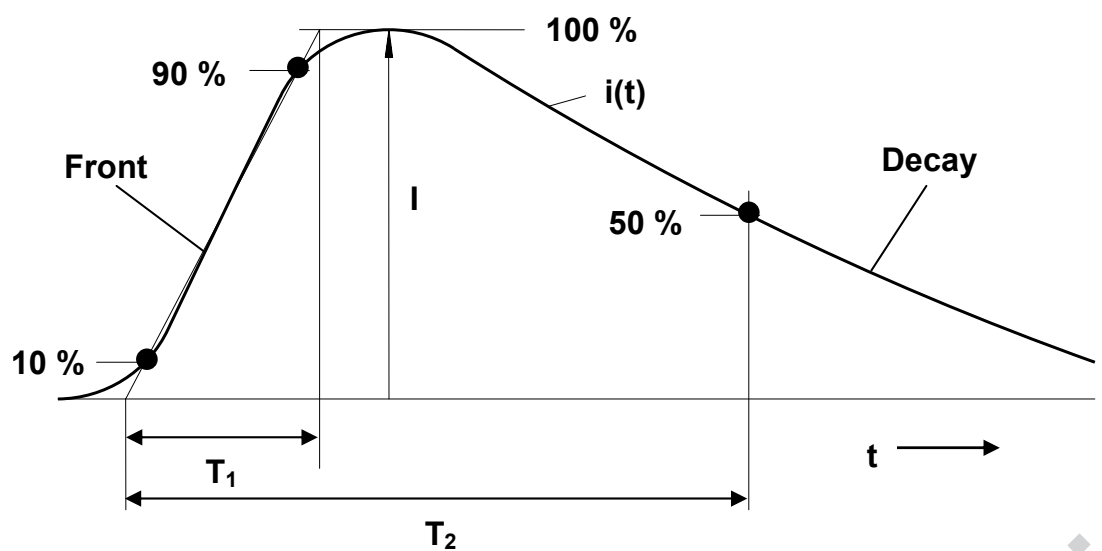

Fig. 1 Standard waveform suggested for approximating lightning current according to IEC 62305.

shows the charge of each waveform if the maximum current of the waveform was $1 \mathrm{kA}$. The two shapes are very similar, but the biggest difference is how smoothly they rise starting at time $=0$. The difference does not dramatically affect the overall shape nor the integral (i.e. - charge) of each type of waveform, but strongly influences the derivative. The waveforms are shown in Figure 2 and the corresponding derivative of each waveform is shown in Figure 3 . It is noted that the $T_{1}$ specified parameter strongly affects the magnitude of the $d i / d t$. For example, the maximum di/dt of the $0.25 / 100$ waveform is several hundred times greater than the maximum di/dt of the 100/1000 waveform. Depending on the mathematical representation chosen, the maximum $d i / d t$ for a single waveform can vary by approximately an order of magnitude. The equation for induced voltage is $V=L d i / d t$, so the voltage calculated is directly proportional to the derivative. Using one equation vs the other to model the IEC 62305 waveform can result in dramatically different voltage calculations. This observation means that simulated voltage results are highly sensitive and variable. The consequence of this sensitivity is that a small change in the simulation parameters can have a large impact on results. When combined with the fact that a lightning strike can have Megavolts of potential that the simulation does not account for, it is not possible to rely on voltage results as accurately representing values found in actual lightning strikes. We therefore do not focus on simulating voltage and do not report voltages calculated. To model an arc, we previously used a voltage-dependent switch set at $25 \mathrm{kV}$. The problem with this approach is it makes the charge measured through the arc very sensitive to voltage when it is not possible to trust the voltage modeled at the switch. Our simulations set the switch threshold to a small non-zero nominal value of $1 \mathrm{kV}$, which ensures the current is only measured when there is a substantial amount of voltage. Note that two switches are used with opposite polarity so that a positive or negative voltage will activate the switch.

\begin{tabular}{|c|c|c|c|c|c|c|c|c|c|}
\hline \multicolumn{2}{|c|}{ Specified parameters } & \multicolumn{3}{|c|}{ Double Exponential } & \multicolumn{4}{c|}{ Heidler } \\
\hline$T_{1}(\mu s)$ & $T_{2}(\mu s)$ & $i_{\max }$ & $\alpha$ & $\beta$ & Charge & $i_{\max }$ & $\alpha$ & $\beta$ & Charge \\
\hline 0.25 & 100 & 1006.24 & $9.78 \mathrm{e}-08$ & 0.000143 & 0.144 & 1008.09 & $4.57 \mathrm{e}-07$ & 0.000143 & 0.144 \\
\hline 1 & 200 & 1011.32 & $3.98 \mathrm{e}-07$ & 0.000284 & 0.286 & 1014.80 & $1.83 \mathrm{e}-06$ & 0.000284 & 0.287 \\
\hline 10 & 350 & 1055.50 & $4.34 \mathrm{e}-06$ & 0.000468 & 0.487 & 1076.34 & $1.91 \mathrm{e}-05$ & 0.000475 & 0.488 \\
\hline 100 & 1000 & 1224.82 & $5.35 \mathrm{e}-05$ & 0.00112 & 1.16 & 1318.09 & 0.000206 & 0.00119 & 1.12 \\
\hline
\end{tabular}

Table 1 Waveform function parameters calculated for specified waveform parameters

The choice of equation has little effect on the charge in the waveform. Thus it is the charge that is the simulation result that we focus on in this paper. The waveform parameters chosen for the IEC 62305 waveform do have a strong effect on the total charge of the waveform. IEC 62305 and other sources suggest different values for different types of lightning strokes ranging from $0.25 / 100$ to $100 / 1000$ ( $\mu s$ ) depending 


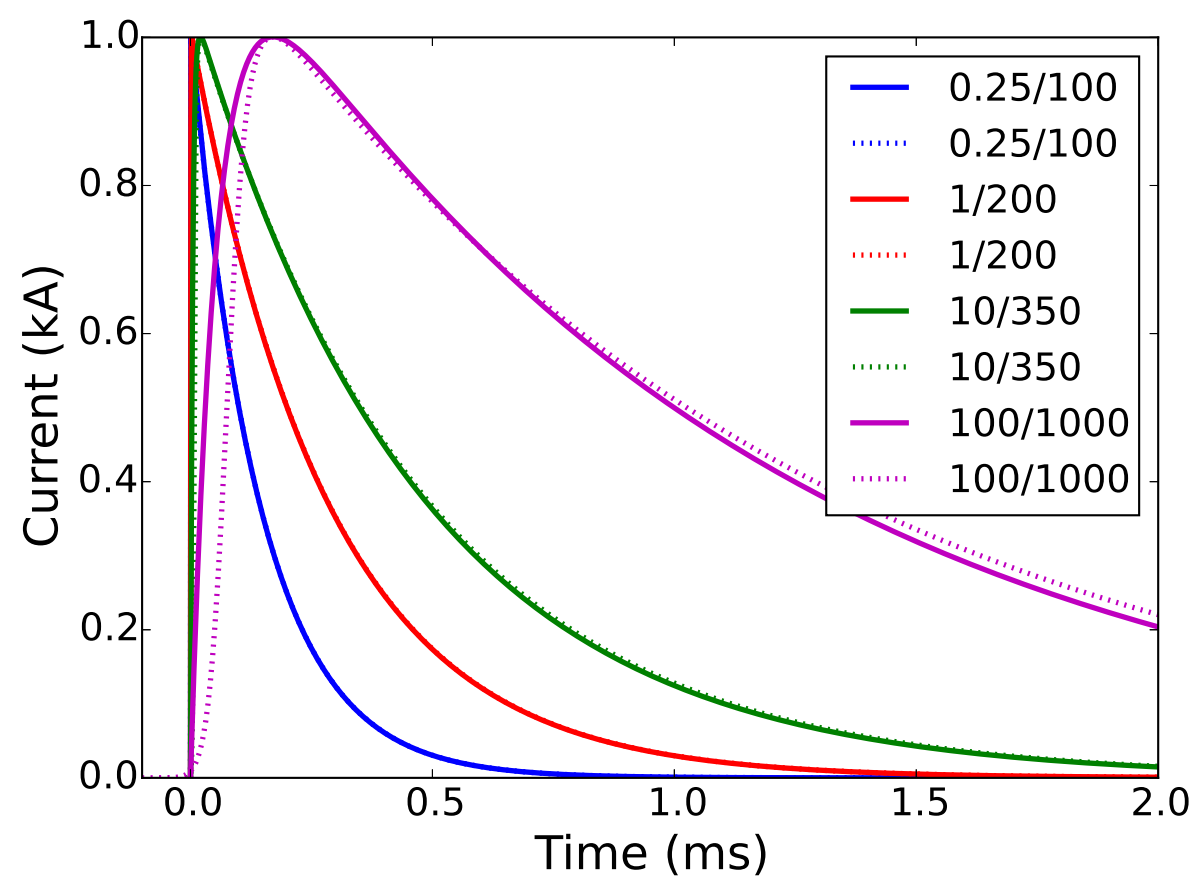

Fig. 2 Example waveforms by specified parameters. Solid lines are modeled with a double exponential and dashed lines with the Heidler equation.
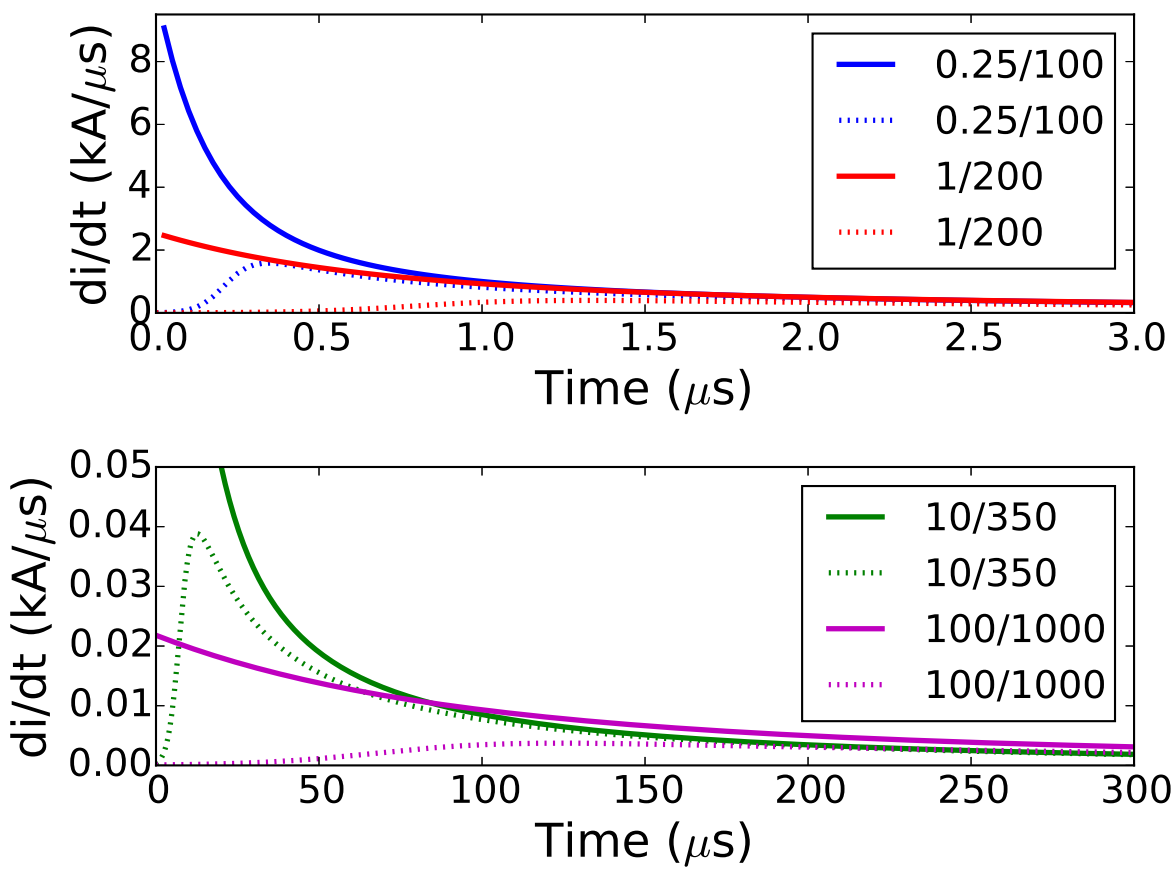

Fig. 3 Derivative of example waveforms by specified parameters and approximating function. Matches functions plotted in Figure 2. 


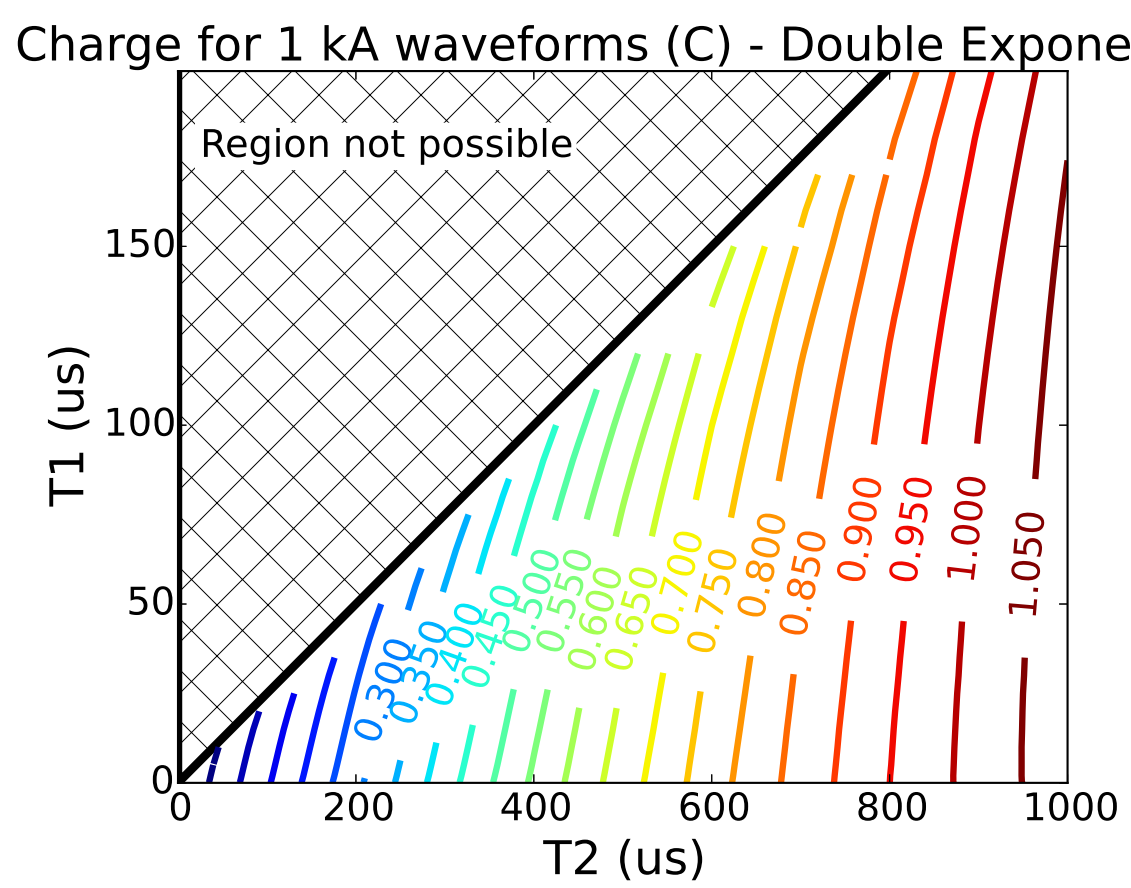

Fig. 4 Total charge in waveform for the double exponential function.

on the lightning stroke. In Figure 4 we plot $T_{1}$ and $T_{2}$ on the $\mathrm{y}$ and $\mathrm{x}$ axes respectively and show charge for a waveform with a peak current of $1 \mathrm{kA}$ on a color scale from blue to red. The total charge is strongly related to the value $T_{2}$. This means that choosing to use a smaller $T_{2}$ for a simulation will give results that are less likely to indicate a hole will be formed. The charge in the waveform is linearly dependent on the maximum current for a given $T_{1}$ and $T_{2}$, so it is possible to quickly calculate the charge in a waveform with higher maximum current. For example, the 100/1000 waveform with $1 \mathrm{kA}$ has approximately $1 \mathrm{C}$ of charge - if a 100/1000 waveform had a $10 \mathrm{kA}$ max current the charge would be $10 \mathrm{C}$ or if it had a $100 \mathrm{kA} \max$ current the charge would be $100 \mathrm{C}$.

The problem with the IEC waveform in general can be summarized using Bazelyan and Raizer's words that standard waveforms were "intended for currents of laboratory sources simulating lightning, rather than for natural lightning" [4]. It is likely the case that these waveform parameters have persisted because equipment can replicate them and not because they faithfully represent lightning strikes. An article from 1962 describes this pattern, "To reconcile a natural phenomenon with an experiment in the laboratory is a difficult task, as is shown by the history of lightning research. Evaluations and knowledge obtained by model tests on a small scale are often incorrect, but survive for a remarkably long time" [21]. It is important to recognize that the waveform used in a simulation is an assumption and variation should be accounted for.

The shape and intensity of actual lightning current depends on a wide variety of factors such as conductivity of the air, height of clouds, size of clouds and many more which make the waveform highly variable [29]. Lightning researchers have taken measurements to empirically characterize lightning waveforms. Figure 5 shows several examples of lightning current waveforms that were measured. It is readily apparent that they are all very different and none of them precisely match the waveform specified in IEC 62305. These waveforms simply do not fit one particular shape or function. One way to characterize real-world lightning is to use a collection of such waveforms to identify the distribution of a variety of parameters describing the waveforms. A recent publication put forth distributions for measured parameters of lightning waveforms [11]. We include several of those parameters relevant to our paper in Table 2.

The charge is the integral of the current, which not only depends on the maximum current, but also the duration of the current. It may be possible to have two flashes with the same peak current but very different total charge. The nature of positive flashes results in strikes that generally have higher currents and more charge, but they make up about $10 \%$ of cloud to ground strikes, with the rest being negative 


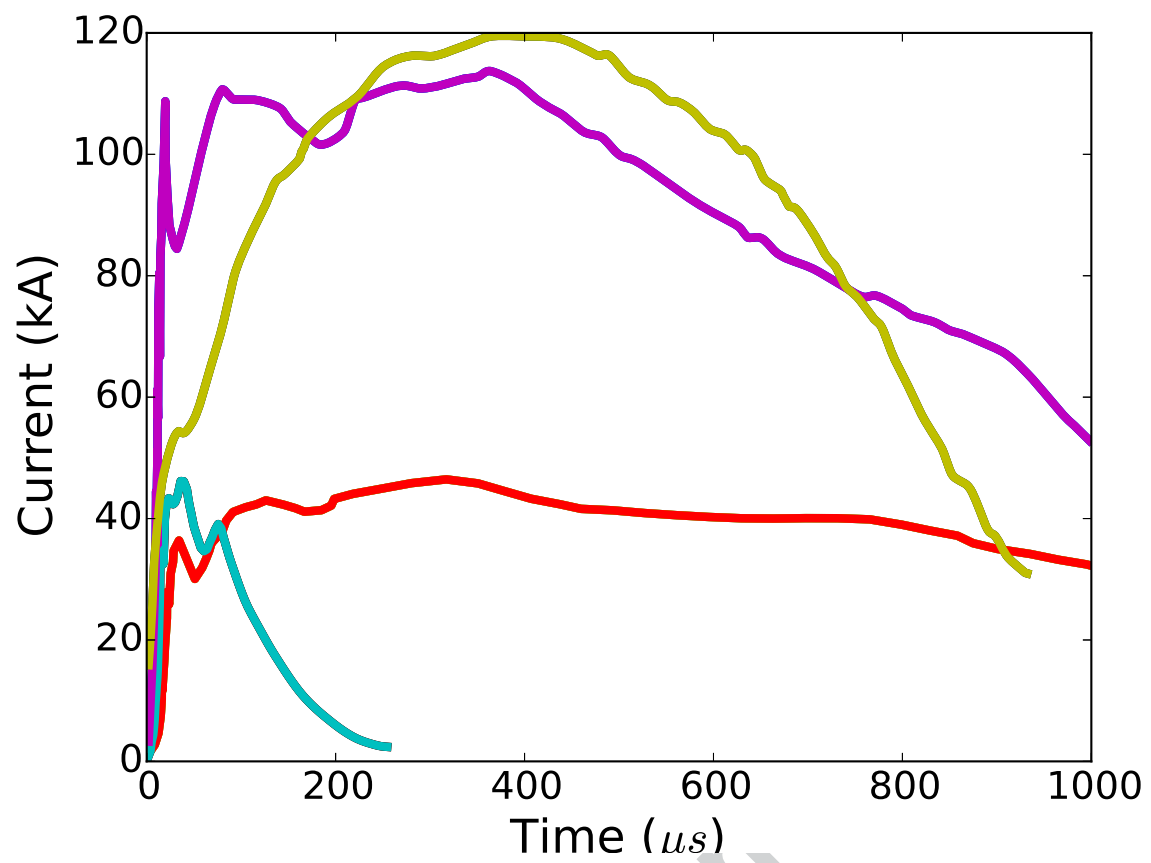

Fig. 5 Examples of measured positive and negative lightning waveforms, all made positive. Adapted from Heidler et. al [16].

\begin{tabular}{|l|c|c|c|}
\hline Waveform & Max Current $(\mathrm{kA})$ & Max di/dt $(\mathrm{kA} / \mu s)$ & Charge (C) \\
\hline Negative flashes & \multicolumn{3}{|l|}{} \\
\hline Top 50\% & 30 & 100 & 20 \\
\hline Top 1\% & 150 & 400 & 200 \\
\hline Positive flashes & \multicolumn{3}{|l|}{} \\
\hline Top 50\% & 35 & 100 & 80 \\
\hline Top 1\% & 500 & 400 & 700 \\
\hline
\end{tabular}

Table 2 The $50 \%$ and $1 \%$ largest values taken from distributions for measured parameters of lightning waveforms [11].

flashes. To provide context, a survey over an 8 year time period found an average of 15.9 million strikes. That number would result in approximately $15,9001 \%$ positive flashes and 143,000 1\% negative flashes $[24,25,17]$.

We use the four different waveform parameter sets mentioned previously that are found in IEC 62305: $0.25 / 100,1 / 200,10 / 350$ and $100 / 1000$. We also vary the impedance values of some materials that are not precisely known to include a tolerance and to measure the sensitivity of the output to the input values. Lastly we vary where the lightning enters the structure.

\subsection{Conductor properties}

A circuit was used to model a house where a fire recently took place at the same time lightning struck. It was determined that the fire was caused because the lightning current caused a hole in the CSST. We ran the simulations with and without a bonding wire to show the effect of the bonding wire on the creation of a hole. We modeled several different locations lightning current could have entered the home as done in [15]. The scenario is shown in Figure 6. The vertical line represents a number of possible conductors that can be found in a house that are not well-grounded. The dashed line represents the hypothetical bond wire that was not present when lightning hit the home. Note that current \#5 could enter the top of the house as in the case of lightning current entering through the chimney or current \#5 could enter at the base of the house as in the case of lightning current entering through piping. 


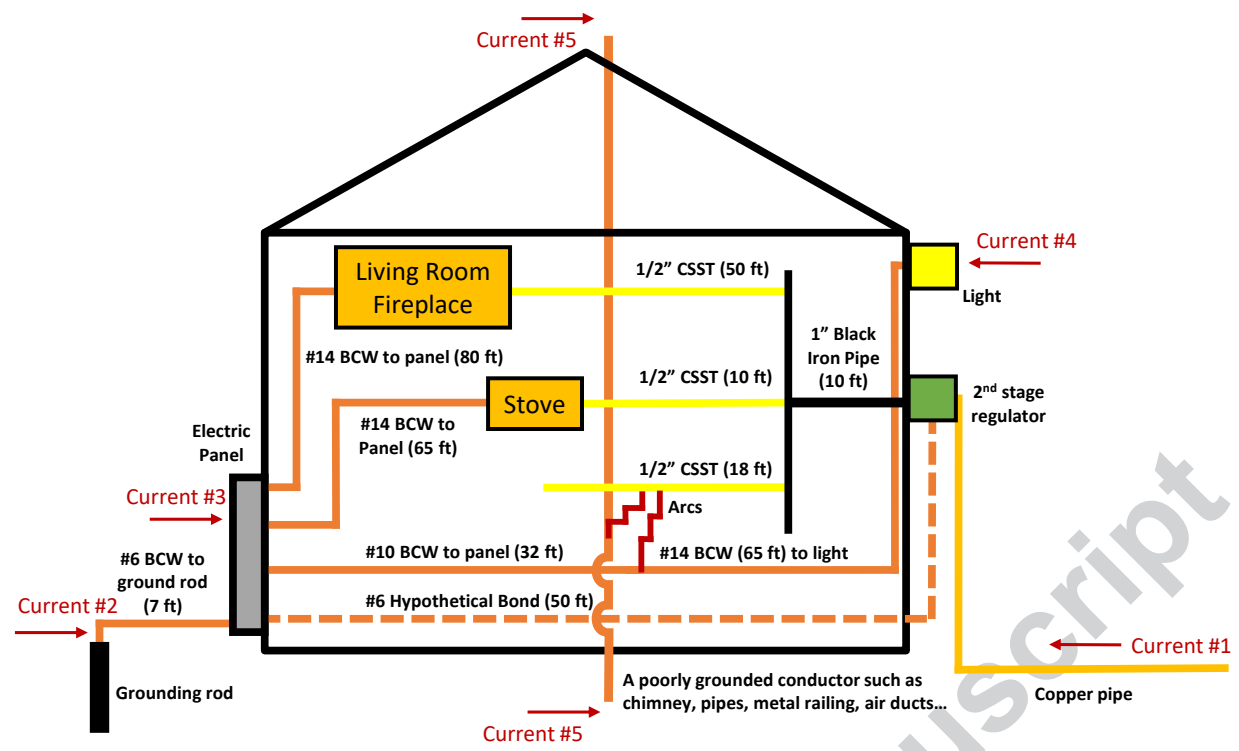

Fig. 6 Scenario derived from recent fire caused by lightning current on CSST.

The impedance values of the various electrical conductors were calculated based on the lengths of the conductors measured after the fire and the impedances per unit length of such conductors. Table 3 shows the resistance and inductance values of the different materials per unit length. The copper wiring are all standard American Gauge Wire (AWG) sizes with well-established resistances [9]. The wire diameters are also well-known [32] and using the equation below the inductance for a straight wire can be calculated: $[12,31]$

$$
L=0.002 l\left[\ln \left(\frac{2 l}{d}-0.75\right]\right.
$$

where $\mathrm{L}$ is the inductance in $\mu \mathrm{H}, \mathrm{l}$ is the length of the wire in $\mathrm{cm}$ and $\mathrm{d}$ is the diameter of the wire in $\mathrm{cm}$. The CSST resistance and inductance are estimated based on measurements from previous reports $[14,33]$.

\begin{tabular}{|l|c|c|}
\hline Material & $\Omega / 1000 \mathrm{ft}$ & $\mu H / 1000 \mathrm{ft}$ \\
\hline AWG \#6 & 0.395 & 768.0 \\
\hline AWG \#10 & 0.999 & 796.3 \\
\hline AWG \#14 & 2.52 & 824.6 \\
\hline CSST & 20 & 300 \\
\hline
\end{tabular}

Table 3 Resistance and inductance values per length of different gauge wires and CSST.

The circuit schematic used for the simulation is shown in Figure 7 with the impedance values from Table 3. The resistance of the electrical grounding rod was measured after the fire and the inductance is an estimate from papers and prior reports $[15,14]$. The impedance value of the black iron pipe was unknown, so we based our estimate on CSST. It is common to have CSST at this location in homes, so modeling CSST here is not unrealistic. The impedance of the gas line is unknown, but we reasoned that it would be significantly more than the electrical ground since the ground rod is designed to have low impedance. We therefore selected a value 2 times the impedance of the grounding rod.

We ran simulations for every combination of parameter values found in Table 4 . We varied the impedance values of the three lengths of CSST, the electrical ground and the black iron pipe by $25 \%$ since the impedance per length is difficult to measure precisely and the lengths are not precise. In reality the length of these components can differ from structure to another by much more than $25 \%$. We tried each combination of these 6 values varied in two ways: $+25 \%$ and $-25 \%$. That gave $2^{6}=64$ combinations, which was combined with the $4 * 5 * 2=40$ combinations of entrance, waveform and bonding to give 2560 total simulations. The maximum current value was $10 \mathrm{kA}$. Because the results scale linearly with maximum current on any 


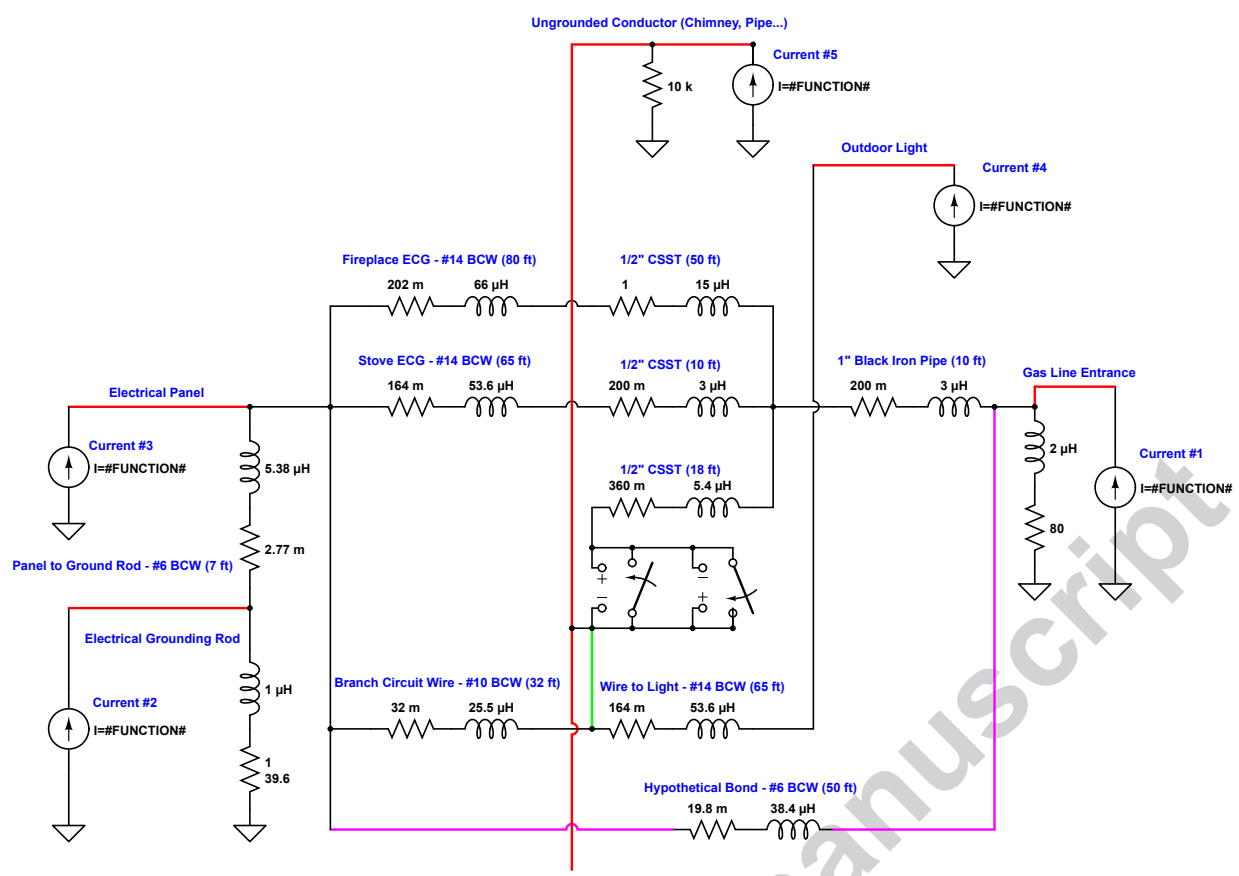

Fig. 7 Circuit schematic to run simulations. Red wires were toggled in different simulations to allow only one current source at a time. The green wire was present when using current sources 1-4 and not for current source 5 to properly model the switch. The magenta wire was toggled to include or exclude the grounding bond wire.

given line, results can be calculated for any maximum current. For example, to calculate the result for a maximum current of $30 \mathrm{kA}$, the median for a negative strike, one would multiply the result by 3 . For a maximum current of $150 \mathrm{kA}$, representing the top $1 \%$ of strikes, one would multiply by 15 .

\begin{tabular}{|l|c|c|c|}
\hline Time parameters $\mu s / \mu s)$ & Entrance & Impedances & Bond \\
\hline $0.25 / 100$ & 1 & $+/-25 \%$ & Yes \\
$1 / 200$ & 2 & & No \\
$10 / 350$ & 3 & & \\
$100 / 1000$ & 4 & & \\
\hline
\end{tabular}

Table 4 Parameters varied for circuit simulations.

\section{Results}

The results from all 2560 simulations are seen in Figure 8 where each data point represents one simulation. The y axis shows the simulations in groups by the waveform parameters used $(0.25 / 100,1 / 200,10 / 350$ or $100 / 1000)$ and the location where lightning entered the structure in the simulations given by numbers $(1$, 2, 3, 4 or 5) corresponding to numbering in the Figure 6 . Each black point is a simulation without bonding and each red point is a simulation with bonding. Different points for each color on each line are different combinations of impedance values that were varied by $25 \%$.

A vertical dashed line is placed at $0.1 \mathrm{C}$, which is the threshold for creating a hole in the CSST. This threshold is for the 1 billion feet of yellow jacketed CSST installed in the United States (more recent CSST with a black jacket has a higher threshold). For bonding to prevent the creation of holes, all red data points would need to be to the left of the $0.1 \mathrm{C}$ vertical line. For simulations where lightning entered at location 2 or 3 , some had results to the left of the dashed line (indicating charge less than $0.1 \mathrm{C}$ ) and some results to the right of the dashed line (indicating charge greater than $0.1 \mathrm{C}$ ). For locations 2 and 3 , the results indicate that depending on the variance of the impedance parameters the result may indicate a hole or not. 


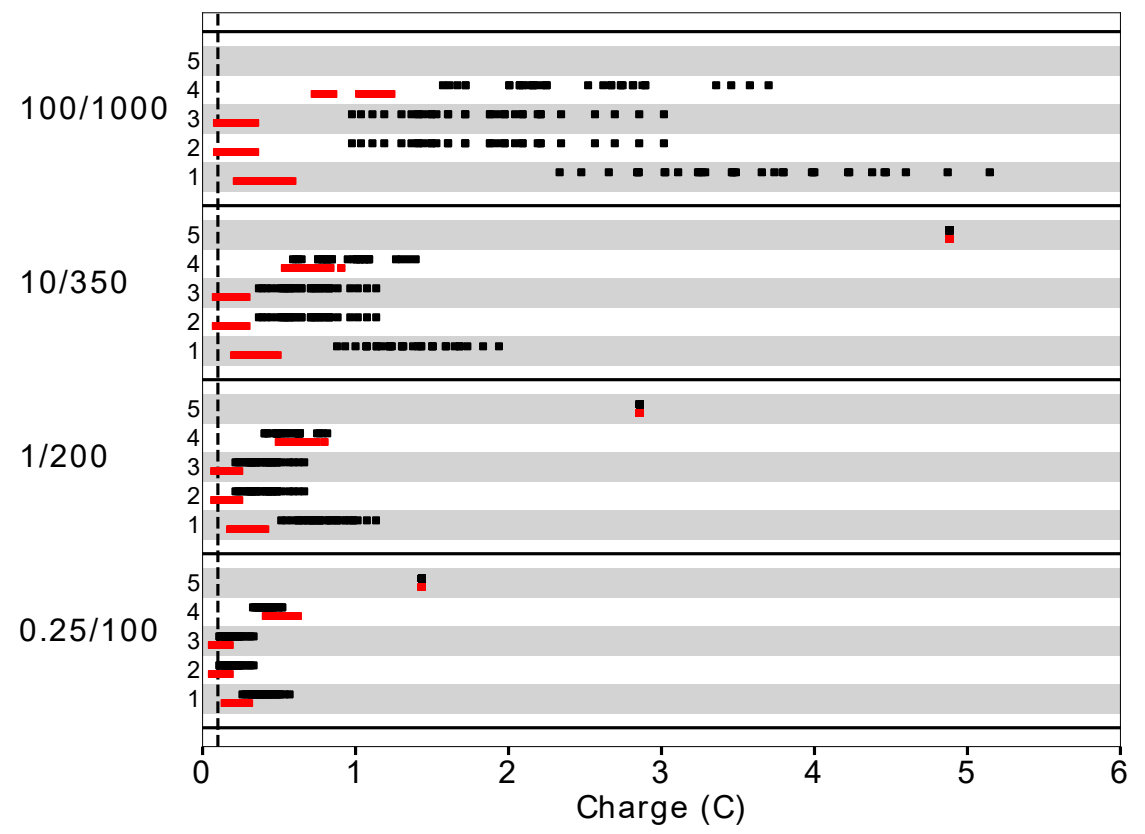

Fig. 8 Results from all 2560 simulations where black points represent simulations without bonding and red points represent simulations with bonding. The waveform parameters used and the location number where lightning entered are labeled on the $\mathrm{y}$-axis.

For simulations where lightning entered at location 1, 4 or 5 all results were to the right of the dashed line, indicating that no matter how the parameters were varied, a hole would always have occurred.

We note that these results are for a $10 \mathrm{kA}$ max current waveform, which is well below the median. To get results for the median all values should be multiplied by 3 (corresponding to the waveforms having a maximum current value of $30 \mathrm{kA}$ ); to get the top $1 \%$ values should be multiplied by 15 . Figure 9 shows results for input waveforms with a peak current of $30 \mathrm{kA}$ and all of the red data points are to the right of the threshold. For input waveforms with a peak current of $150 \mathrm{kA}$ the red data points are all well to the right of the threshold. For safety to be assured, all red points must be to the left of the dotted line.

There are several patterns in the results to highlight. First, the greater the value of the T2 waveform parameter (larger T2 parameters are found higher up the y-axis), the greater the charge through the arc. This is likely because a larger T2 means more total charge in the waveform. It can also be seen that when lightning entered through entrance 1, 2 or 3, the charge was similar because all three entrances are connected via low impedance to the electrical ground. For entrances 4 and 5 the charge was significantly higher. For entrance 5 there is no variance because the only path to ground is through the CSST and therefore all of the charge goes through the arc. For this reason the red data point is directly over the black data point and represents the total charge found in the input current waveform.

A more subtle observation is that the difference between the red and black points increases with longer waveforms. This is not merely due to an increase in total charge, but is a result of the larger T1 parameter (larger T1 parameters are found higher up the $\mathrm{y}$-axis). The T1 parameter determines how quickly the waveform rises and therefore determines how much of an effect inductors in the circuit contribute to impedance of the flow of current. The result is that the bond wire has more or less of an effect depending on the other inductance values in the circuit and the rise time of the current waveform. 


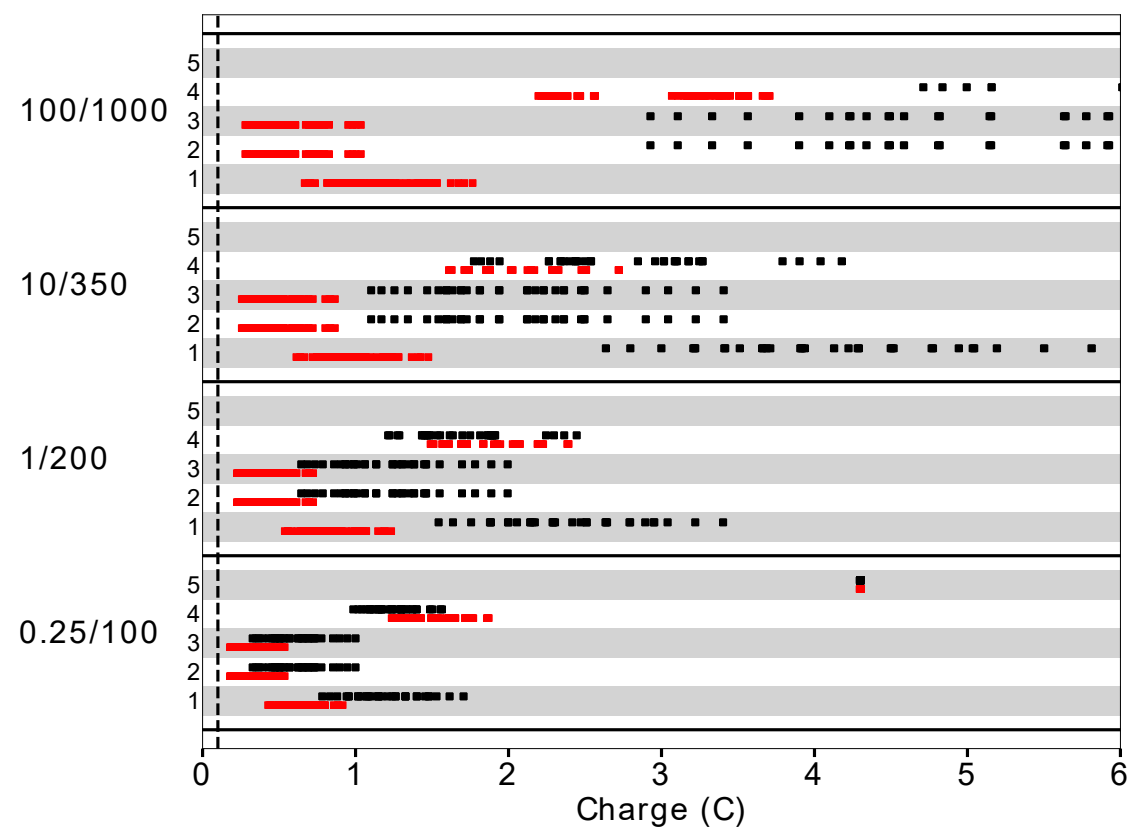

Fig. 9 Results from all 2560 simulations with an input waveform with a peak current of $30 \mathrm{kA}$.

\section{Discussion}

The results show that the charge through the arc calculated from the simulations depends most strongly on the waveform parameters used because those parameters determine how much charge is in the input current waveform and how quickly the current changes. The more charge in the input current waveform the more charge will go through the arc. The waveform parameters are a modeling choice the user makes in setting up the simulation. This choice must be justified to show that it faithfully represents what will occur in nature. Selecting waveform parameters that result in a small amount of charge underestimates the amount of charge in lightning and can produce results that indicate the material will resist perforation while in realistic conditions perforation would occur. Doing this makes something look safe when in reality it is not.

The charge through the arc is also strongly dependent on where lightning enters the structure, which reaffirms what was previously shown in [15]. The charge is much larger when the lightning current does not enter through the gas line or the electrical line. This means significantly more charge will go though the arc if the lightning enters through a chimney, metal plumbing pipes, HVAC equipment, outdoor lighting and so forth. The increase in charge means a perforation is more probable and therefore a fire is much more likely.

The charge through the arc also depends on the relative impedance values of different materials in the structure and the waveform shape, which was also shown previously in [15]. In the present work we demonstrate how to put tolerances on different impedances and calculate a range of results by computing every combination maximum and minimum of the tolerances. We used 6 different sets of values resulting in 64 different combinations. The simulations we performed each took a few seconds on a single processor. Assuming each simulation took 3 seconds, an 8 core machine could perform 230,400 simulations in a 24 hour period, which would correspond to 17-18 simulation parameters for which every combination of a pair of maximum and minimum tolerance values could be simulated.

This work also showed that simulations of voltage cannot faithfully represent real situations because small changes in the mathematical representation of what is considered the same waveform has a strong effect on the voltages simulated. When this result is viewed with the fact that a real lightning strike packs 
orders of magnitude higher voltage than the arc threshold for the CSST, it is clear that sufficient arc voltage can occur easily even in inductively coupled situations. By attempting to model the arc, it is easy to get simulation results where the voltage does not meet the arc threshold, leading to the erroneous conclusion that no hole could be formed. Such a result masks the fact that the voltage result is highly dependent on the simulation waveform and does not account for the excess voltage present in the lightning strike.

This work also shows that there are dramatic differences in amounts of charge (in addition to voltage) for different waveform parameters. Figure 4 can be used to look at charge in waveforms with different parameters. This work makes it clear why it is no surprise that simulations using shorter waveforms conclude a hole would not be formed. The waveform parameters must be justified and not merely assumed. This includes waveforms that have been in use for decades, but do not faithfully represent what happens in nature. We believe that the four waveforms we used are not enough to approximate the breadth of lightning current waveforms found in nature and one area of future research is to develop a set of waveforms that could be trusted to match natural conditions. The waveforms set in IEC 62305 from decades ago were created because laboratory equipment could replicate such waveforms and not because they faithfully represent nature. It is now technically possible to rapidly simulate a wide variety of waveforms. The practice of simulating variation should be adopted when analyzing how a fire started, in design of products and in design of safety systems. Another area of future research would be to compile a set of measured lightning waveforms to use as the simulation waveform instead of relying on mathematical approximations.

Another area of research is to better define the relationship between maximum current and total charge. The research we have cited shows statistics for each parameter individually, but not the dependence between the two. Some assume that a higher maximum current means that the charge will be higher, but it is possible to have a smaller maximum current and a longer waveform that results in a higher charge. This is particularly important because it is possible to measure maximum current using resources like the National Lightning Detection Network (NLDN). If the particular strike can be identified from the NLDN report, the maximum current could be used as an input to a simulation. The problem is that the charge of the strikes is not known from the NLDN. One source showed the relationship between maximum current and length of continuing current [27], but more data is needed. If many strikes that do not appear strong have long durations they could be more dangerous. Some strikes may have duration in hundreds of milliseconds. One limitation of our study is we only performed simulations up to $2 \mathrm{~ms}$. So our results may underestimate total charge in these cases.

Finally, we note that there may be other ways to infer circuit parameters for a specific case. One example is using the size of a hole in CSST to estimate the amount of charge that caused the hole. The science behind this relationship was studied by Hagenguth more than 60 years ago [13]. The challenge is determining how well the science translates to the specific case of CSST. For example, CSST is corrugated and has a dielectric jacket that may focus the arc to the metal. Hagenguth did not study these factors. So the variance of these and any other factors must be taken into account. The overall guiding principle is that for any value that is assumed or inferred, a tolerance of what the values could be must be justified and the maximum and minimum tolerance values should be simulated.

Our work shows that it is easy to find a set of simulation parameters that will support or contradict a given opinion, but what matters is that results are consistent across a set of simulation parameters that will occur in nature. For the manufacturer's requirement of bonding, it is easy to get simulation results from a few simulations showing bonding will prevent fires if the waveform used has a total charge well below the median strike, has carefully selected impedance values and/or the circuit is setup to ensure the switch modeling an arc will not be activated. To summarize, the problem is not to find one or two simulations that support an opinion, but to show for all realistic parameters that the damage will not happen. That is the key to safe codes and standards. We are unaware of anybody doing so when analyzing the manufacturer's data to make sure all reasonable sets of simulation parameters would give the same result. Our work shows that if that had been done, the claims made by the manufacturers would not be accepted by the Code writing authorities.

\section{Conclusions}

Circuit simulations are a powerful tool for estimating effects of lightning on CSST and predicting fires, but they must be used carefully. All simulation parameters are based on facts or assumptions. We found that changing parameters of the current waveform to simulate lightning had a significant effect on the outcomes of the simulations. We also found that the results depend on other parts of the circuit such as where the 
lightning enters and what the impedance values of the circuit are. We draw several conclusions from these results:

1) The first is that if a parameter cannot be established as fact, a range of values should be used. As an example, even though in specific cases the peak current of the lightning strike can be estimated, if the waveform shape is not known, then a range of waveforms should be used. This is critical in order to be confident the simulation results are applicable to natural lightning strikes.

2) The second conclusion is that a combination of parameters must be tested to ensure that all cases result in the same conclusion. For example, it would not be conclusive to run a set of simulations with one waveform while varying lightning entry points and then one entry point and a set of varying waveforms. All combinations should be simulated because of the unique dependencies.

3) The third conclusion is that using simulations to estimate voltages is too variable to provide reliable results in the case of simulating voltages inside a structure due to a lightning strike.

These conclusions demonstrate that in order to trust simulation results, assumptions must be justified and uncertainties modeled through combinations of simulation parameters. Our results support that it may be possible to produce a simulation that shows bonding will prevent a fire for a specific case, but that such simulations are the exception rather than the rule when considering all uncertainties. This work has important implications for drawing conclusions from circuit simulations and ultimately protecting homes where CSST is installed.

\section{Funding Statement}

This study was funded in its entirety by the authors in the interest of fire safety. 


\section{References}

1. Ordinance 2nd Reading - Building Inspection: Consider Ordinance 2016-O0075 amending Chapter 28, Article 28.15 of the Code of Ordinances of the City of Lubbock with regard to the adoption of the 2012 International Fuel Gas Code with local amendments., 2016.

2. American Gas Association. Interior Fuel Gas Piping System Using Corrugated Stainless Steel Tubing. ANSI/AGA LC-1-1991. American National Standards Institute, 1991.

3. R.B. Anderson and A.J. Eriksson. Lightning parameters for engineering application. Electrica, 69:65$102,1980$.

4. Eduard M. Bazelyan and Yuri P. Raizer. Lightning Physics and Lightning Protection. CRC Press, Boca Raton, FL, 2000

5. K. Berger, R.B. Anderson, and H. Droninger. Parameters of lightning flashes. Electra, 41:23-37, 1975.

6. Kenneth L. Cummins, E. Philip Krider, and Mark D. Malone. The u.s. national lightning detection networktm and applications of cloud-to-ground lightning data by electric power utilities. IEEE Transactions on Electromagnetic Compatability, 40(4), 1998.

7. Kenneth L. Cummins and Martin J. Murphy. An overview of lightning locating systems: History, techniques, and uses, with an in-depth look at the u.s. nldn. IEEE Transactions on Electromagnetic Compatability, 51(3), 2009.

8. E. Brian Curran, Ronald L. Holle, and Raul E. Lopez. Lightning casualties and damages in the united states from 1959 to 1994. Journal of Climate, 13:3448-3564, 2000.

9. J.R. Davis, editor. Copper and Copper Alloys. ASM International, 2001.

10. Robert A. Durham and Marcus O. Durham. Does corrugated tubing + lightning = catastrophic failure? IEEE Transactions on Industry Applications, 48(4):1243-1250, July 2012.

11. William R. Gamerota, Jean O. Elisme, Martin A. Uman, and Vladimir A. Rakov. Current waveforms for lightning simulation. IEEE Transactions on Electromagnetic Compatability, 54(4), 2012.

12. Frederick W. Grover. Inductance calculations: working formulas and tables. Dover Publications, 2009.

13. J.H. Hagenguth. Lightning stroke damage to aircraft. Transactions of the American Institute of Electrical Engineers, 68, 1949.

14. Andrew Hammerschmidt and Christopher J. Ziolkowski. Validation of installation methods for CSST gas piping to mitigate indirect lightning related damage. Technical report, Gas Technologies Institute, 2013.

15. Bryan Haslam, Donald Galler, and Thomas W. Eagar. Fire safety of grounded corrugating stainless steel tubing in a structure energized by lightning. Fire Technology, 52(2):581-606, 2016.

16. F. Heidler, W. Zischank, Z. Flisowski, Ch. Bouquegneau, and C. Mazzetti. Parameters of lightning current given in IEC 62305 - background, experience and outlook. In 29th International Conference on Lightning Protection, Uppsala, Sweden, 2008.

17. Gary R. Huffines and Richard E. Orville. Lightning ground flash density and thunderstorm duration in the continental united states: 1989-96. American Meteorological Society, 1999.

18. International Electrotechnical Commission. IEC 62305-1: Protection Against Lightning. American National Standards Institute, 2010.

19. Lightning Protection Institute. NFPA 54 (National Fuel Gas Code) to include bonding requirements. LPI Tech Letter, 10(10), 2008.

20. Martin Uman. Lightning. Dover Publications Inc., 1969.

21. D. Muller-Hillebrand. The protection of houses by lightning conuctors- an historical review. Journal of the Franklin Institute, 274:34-54, 1962.

22. National Fire Protection Agency. NFPA 54: National Fuel Gas Code. 2015.

23. National Fire Protection Agency. NFPA 780: Standard for the Installation of Lightning Protection Systems. 2017.

24. Richard E. Orville. Lightning ground flash density in the contiguous united states-1989. American Meteorological Society, 1991.

25. Richard E. Orville and Alan C. Silver. Lightning ground flash density in the contiguous united states: 1992-95. American Met, 1997.

26. C.E. Pereira, S.C. Snow, and M.M. Dargi. LT-14-3900: Test report of lightning direct effects tests on CSST samples. Technical report, Lightning Technologies, an NTS Company, 2014.

27. V.A. Rakov, A. Borghetti, C. Bouquegneau, W.A. Chisholm, V. Cooray, K. Cummins, G. Diendorfer, A. F. Heidler, Hussein, M. Ishii, C.A. Nucci, A. Piantini, Jr. O. Pinto, X. Qie, F. Rachidi, M.M.F. Saba, T. Shindo, W. Schulz, R. Thottappillil, S. Visacro, and W. Zischank. Cigre technical brochure on lightning parameters for engineering applications. In International Symposium on Lightning Protection 
(XII SIPDA), Belo Horizonte, Brazil, 2013.

28. Vladimir A. Rakov. Fundamentals of Lightning. Cambridge University Press, 2016.

29. Vladimir A. Rakov and Martin A. Uman. Lightning: Physics and Effects. Cambridge University Press, 2003.

30. Dean W. Rivest. Conductive jacket for tubing., 2006.

31. Edward B. Rosa and Frederick W. Grover. Formulas and tables for the calculation of mutual and self-inductance. Technical report, Bulletin of the Bureau of Standards, 1911.

32. S.W. Stratton, editor. Circular of the Bureau of Standards. G, 1914.

33. Michael F. Stringfellow. Validation of installation methods for CSST gas piping to mitigate indirect lightning related damage: Computer simulations of bonding effectiveness. Technical report, PowerCET, 2013.

34. John M. Tobias. The basis of conventional lightning protection systems. IEEE Transactions on Industry Applications, 40(4):958-962, 2004.

35. Martin Uman. The Art and Science of Lightning Protection. Cambridge University Press, New York, NY, 2010.

36. Martin A. Uman and E. Philip Krider. Natural and artificially initiated lightning. Science, 246(4929), 1989.

37. United States Senate. Senate resolution 483: Commending efforts to promote and enhance public safety on the need for yellow corrugated stainless steel tubing bonding, 2012. 